\title{
Understanding the contribution of groove resonance to tire-road noise on different surfaces under various operating conditions
}

\author{
Julien Pinay ${ }^{1, *}$, Yoshinori Saito ${ }^{2}$, Christian Mignot ${ }^{3}$, and Frank Gauterin ${ }^{1}$ \\ ${ }^{1}$ Institute of Vehicle System Technology, Karlsruhe Institute of Technology, 76131 Karlsruhe, Germany \\ ${ }^{2}$ Nihon Michelin Tire Co. Ltd., 163-1073 Tokyo, Japan \\ ${ }^{3}$ Manufacture Française des Pneumatiques Michelin, 63040 Clermont-Ferrand Cedex 9, France
}

Received 13 January 2020, Accepted 21 April 2020

\begin{abstract}
Reducing tire-road noise is now becoming more and more important during the tire development process. Tread profile randomization is used to avoid tonal components and reduce groove resonance noise. To better understand the groove resonance contribution to tire-road noise, we performed acoustic measurements on a test bench with two serial tires. We filled the grooves with acoustic foam to highlight the groove resonance's contribution. We then varied the road surface, the tire load and the driving speed. In the end, we used a multiple linear regression to quantify the interaction between the varying parameters and the groove resonance noise. We show that groove resonance contributes an average of $1.7 \mathrm{dBA}$ to the tire rolling noise of passenger car tires. Groove resonance noise also increases with the driving speed. While the tread pattern and the tire load are responsible for the spectral content of the groove resonance noise, the orientation of the road surface's texture mainly influences the noise level of the groove resonance. The tire manufacturers should carefully consider these findings when developing noise-optimized patterns. This is especially true for tire approval tests, which take place on tracks and usually have a relatively low texture level that is oriented negatively.
\end{abstract}

Keywords: Tire road noise, Groove resonance, Regression, Pavement, Experimental method

\section{Introduction}

Traffic noise and especially tire-road noise is an issue that will increasingly affect people over the coming years. Electro-mobility should namely lead to the disappearance of combustion engine. Tire-road noise will therefore be the highest contributor to vehicle related traffic noise. If we could reduce the rolling noise of passenger cars by 3-4 dBA, approximately 11 billion Euro could be saved each year in the European Union [1]. Different mechanisms contribute to tire-road noise. Besides the noise created by the road excitation and the tread pattern, the groove resonance phenomenon has also been studied for years [2].

Favre showed a good correlation between the measured and calculated groove resonance frequency for different boundary conditions [3]. Saemann compared a slick tire with a ribbed tire and found a difference in rolling noise of $3.5 \mathrm{dBA}$ [4]. Wilken estimated the contribution of one transversal groove of a truck tire to $4 \mathrm{dBA}$ by filling the groove with acoustic foam [5]. Ejsmont et al. compared the influence of several groove geometries (width, angle, asymmetry, microslots, etc.) on tire-road noise using

*Corresponding author: julien.pinay@kit.edu measurements on an outer drum facility with different road surface replica [6]. Fujikawa et al. showed in [7] the influence of road surface absorption on the radiation of groove resonance noise. Fujiwara et al. validated a Finite-Element Model to point out the noise reduction potential using sub-resonators [8]. A static methodology to optimize the pipe network in order to reduce groove resonance noise is presented in [9]. Despite all the research, it is not clear what the real contribution of groove resonance is to tire-road noise for serial tires rolling on real asphalt surfaces. The analyses presented up to now only highlighted the influence of one parameter on the tire rolling noise spectrum. We present a method to quantify the combined influence of the road surface characteristics and the operating conditions on the groove resonance noise. In this study, we isolate the groove resonance noise by filling the grooves with acoustic foam as in [5] and perform noise measurement on an inner drum test bench. We suppose that the noise level difference is entirely due to the foam's effect on groove resonance noise. To understand the influence of the road characteristics, the tire load and the driving speed on the groove resonance, we perform a multiple linear regression using the double lasso method for variable selection. 


\section{Methods}

\subsection{The Inner Drum Test Rig}

The inner drum tire test bench is composed of a drum with a diameter of $3.8 \mathrm{~m}$ and a rigid wheel carrying system. We can drive the tire and the drum separately up to $200 \mathrm{~km} / \mathrm{h}$ [10]. In this study, we want the measurements to take place in free-rolling conditions. Therefore, the drum will always be driving the tire. We fixed the camber and slip angles to $0^{\circ}$ and the room temperature to $20^{\circ} \mathrm{C}$. We kept the inflation pressure constant at 2.5 bar after warming up the tires. We decreased the driving speed from $120 \mathrm{~km} / \mathrm{h}$ down to $30 \mathrm{~km} / \mathrm{h}$ using $2.5 \mathrm{~km} / \mathrm{h}$ steps. Each measurement lasts $10 \mathrm{~s}$ and has a sampling frequency of 44,100 Hz. We used three steps for the tire load: 2400, 3600 and $4800 \mathrm{~N}$. Acoustic panels and acoustic foam are mounted on the test room walls and on the wheel carrying system to prevent noise reflection on the test rig.

We distributed five microphones around the tire (see Fig. 1). Three microphones measured the rolling noise at the tire sidewall according to the CPX method [11]. We also measured the noise at the trailing and the leading edges. However, we could not install these last two microphones at the standardized CPX-position due to the drum curvature. Therefore, we positioned these microphones $20 \mathrm{~cm}$ away from the tire and $15 \mathrm{~cm}$ above the drum surface. To study the possible interaction between all parameters, we performed a full-factorial experimentation plan.

\subsection{Tire design and road surfaces}

The tires studied are a summer and a winter tire, whose tread pattern geometries can be seen in Figure 2. To show the pipe network for each tire and therefore facilitate the results interpretation, we made footprints using ink and paper (see Figs. 2c and 2d). The summer tire's profile consists in four open-ended circumferential grooves. The outer grooves are ventilated towards the tire sidewalls and towards the inner circumferential grooves. One inner groove has resonators with a length of $20 \mathrm{~mm}$. This corresponds to a resonance frequency around $4300 \mathrm{~Hz}$, which should not affect the fundamental resonance frequency of the circumferential groove. The groove network of the winter tire is much more complicated. The grooves namely have a non-prismatic shape and are all ventilated towards the tire sidewalls. The grooves length depend upon their position in the contact patch. Grooves at the leading and trailing edges are much shorter and might not be connected through the contact patch's center. On the other hand, grooves at the contact patch's center are longer and connected to the other grooves.

We filled the tires grooves with acoustic foam to highlight the groove resonance phenomenon. This method has been shown to be efficient for transversal grooves in [5]. We used the Calmflex F-2 foam sheet with a thickness of $5 \mathrm{~mm}$. Because of their too narrow width, we could not fill the transverse grooves of the summer tire with foam. By filling the summer tire's groove with foam, we added a mass of $7.5 \mathrm{~g}$ to the tire, which is distributed over the tire's circumference. As the summer tire weighs $8552.4 \mathrm{~g}$, this

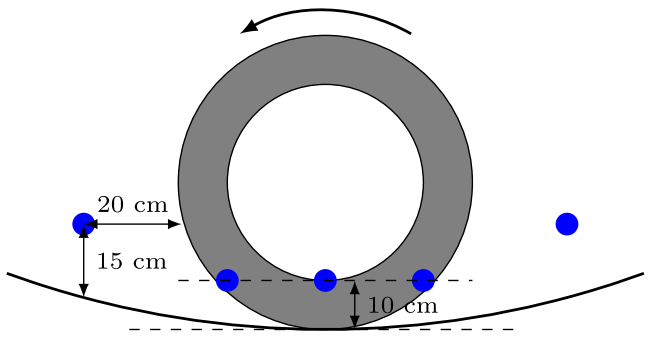

(a) Side view

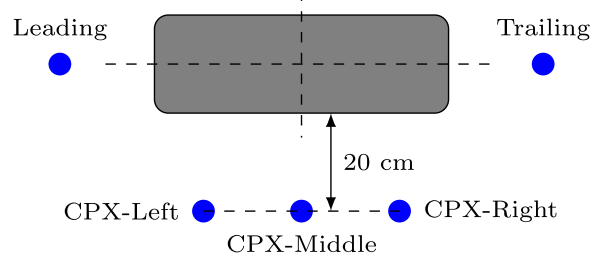

(b) Top view

Figure 1. Microphone positions on the inner drum test bench. The thick arrow at the top of the diagram describes the standard direction of rotation.

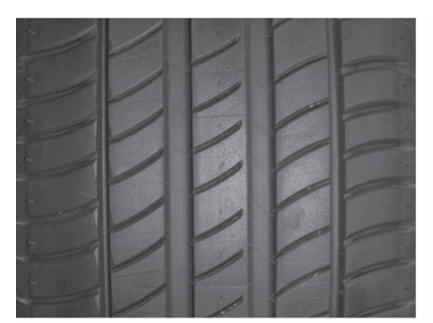

(a) Summer

(c) Summer - Footprint

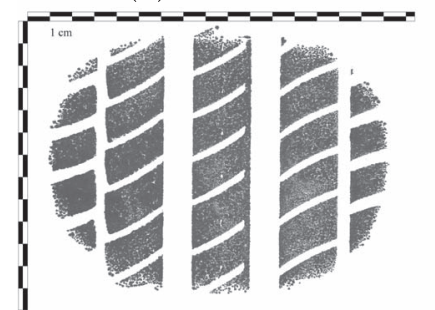

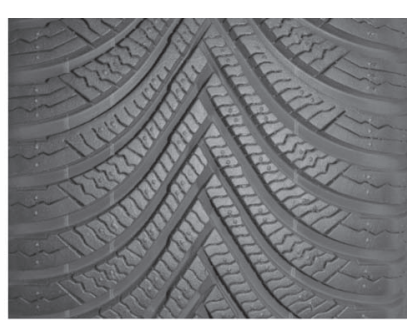

(b) Winter

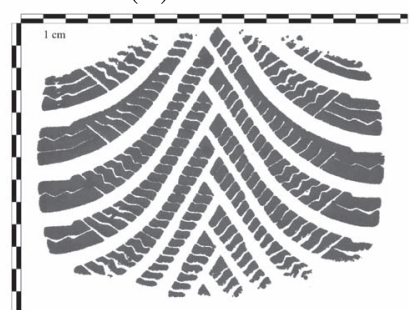

(d) Winter - Footprint
Figure 2. Selected tires for this study. Both tires have the same dimension 205/55R 16 . We made the footprints with a tire load of $3600 \mathrm{~N}$.

corresponds to a mass increase of less than $0.1 \%$. We therefore make the assumption that the foam insert will not have a strong influence on the belt vibrations. We glued the foam only at the bottom of the grooves without touching the tread blocks in order not to affect the stiffness of the tread pattern.

To study the interaction between the road properties and the groove resonance noise, we need to have a broad spectrum of road surface roughness. This is why we chose a smooth asphalt concrete $0 / 5$ (Fig. 3a) and a rough exposed aggregate cement concrete with chippings up to $16 \mathrm{~mm}$ 


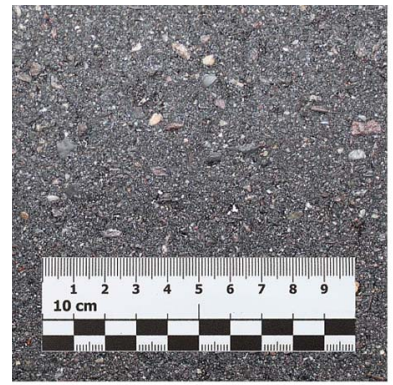

(a) Asphalt 0/5

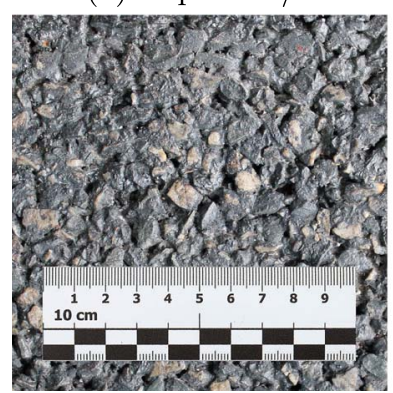

(c) SMA $0 / 8$

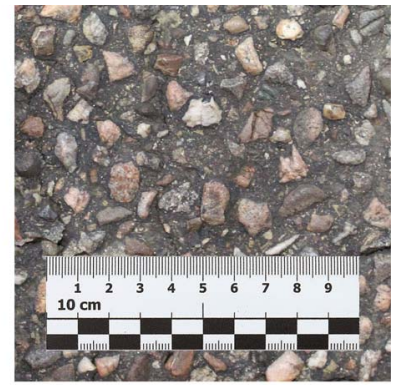

(b) Asphalt 0/11

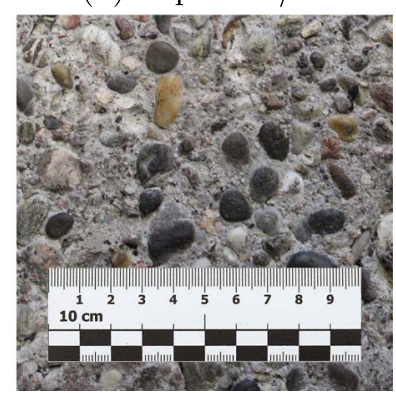

(d) Concrete 0/16
Figure 3. Selected road surfaces for this study.

(Fig.3d). We also tested two surfaces commonly built on national roads in Germany: an asphalt concrete 0/11 (Fig. 3b) and a stone-mastic asphalt (SMA) 0/8 (Fig. 3c). The whole drum surface was scanned using a laser point sensor MicroEpsilon ILD 2300-50. We set the measurements parameters in order to have a longitudinal resolution of $50 \mu \mathrm{m}$. We computed several parameters from the laser measurements to describe the road surface roughness:

- The MPD according to ISO 13473-1 [12];

- The RMS, RSK and RKU according to ISO 13473-2 [13].

MPD is the standard parameter used to describe the roughness of road surfaces when investigating tire rolling noise. RSK and RKU are related to the distribution function of the road surface roughness. RSK describes the asymmetry of the distribution function. A negative skewness consists in a negative oriented roughness: "a flat road with dents". A positive skewness has a positive-oriented texture, which can be simplified as "a flat road with spikes". A skewness of zero is a normal distribution.

RKU measures the peakiness of the roughness distribution function. A normal distribution has a kurtosis of three. If the distribution is peakier (the roughness amplitudes are concentrated around the mean value), then the kurtosis is greater than three. On the contrary, if the distribution function is flat, then the kurtosis is lower than three. The physical meaning of RSK and RKU for the characterization of road surface roughness is well depicted in [14].

The acoustic absorption was measured in-situ with an impedance tube, according to ISO 13472-2 [15]. For the statistical analysis, we build an absorption coefficient from the
Table 1. Values of the operating conditions chosen for the experimental design.

\begin{tabular}{lcccc}
\hline & $\begin{array}{c}\text { Asphalt } \\
0 / 5\end{array}$ & $\begin{array}{c}\text { Asphalt } \\
0 / 11\end{array}$ & $\begin{array}{c}\text { SMA } \\
0 / 8\end{array}$ & $\begin{array}{c}\text { Concrete } \\
0 / 16\end{array}$ \\
\hline MPD [mm] & 0.37 & 1.15 & 1.19 & 1.44 \\
RMS [mm] & 0.21 & 0.52 & 0.95 & 0.86 \\
RSK [-] & -0.92 & 0.26 & -1.37 & -0.33 \\
RKU [-] & 6.21 & 4.21 & 5.29 & 3.08 \\
$\alpha[\%]$ & 2.7 & 2.3 & 12.2 & 1.0 \\
\hline
\end{tabular}

absorption spectrum at relevant frequencies for the first two groove resonance frequencies. This coefficient consists of the average of the absorption coefficient third-octave bands 800,1200 and $1600 \mathrm{~Hz}$. For the rest of this study, we denote $\alpha$ as the absorption coefficient. The computed values for all describing parameters are summarized in Table 1.

\subsection{Spectral and statistical exploitation of the measurements}

\subsubsection{Exploitation of the acoustic measurements}

The acoustic exploitation is performed with Matlab R2018a. We first applied an A-weighting filter on the raw data before computing the SPL of the tire rolling noise. The noise spectrum was computed using Fourier transformation. We chose the window length in order to have a frequency resolution of $4 \mathrm{~Hz}$. We used a Hanning window with an overlap of $75 \%$.

To highlight the contribution of groove resonance noise to tire-road noise, we considered that the acoustic foam completely absorbed the groove resonance noise. In this study, we depict the groove resonance noise as the arithmetic difference between the SPL without the foam and the SPL with foam, as explained in Equation (1):

$$
\Delta \mathrm{SPL}=\mathrm{SPL}_{\text {without foam }}-\mathrm{SPL}_{\text {with foam }} \text {. }
$$

\subsubsection{Generalized regression model}

We want to identify the parameters that influence the groove resonance noise. To do so, we predict the groove resonance noise using the road surface descriptors described in Section 2.2, the driving speed and the tire load. We perform the statistic analysis with JMP Pro 14.2. We performed a preliminary analysis in order to verify the major assumptions of linear regressions such as normality, homoscedasticity, linearity and absence of correlated errors [16]. Transforming variables using mathematical functions is an efficient technique to best meet the required conditions $[16,17]$. This step led us to transform the absorption coefficient with a log function.

We use a generalized regression model using a shrinkage method to select the best parameters. The statistical significance is computed using a Wald Chi-Squared test. To ensure a consistent prediction of the estimates, we used a shrinkage technique called "adaptive double lasso generalized regression". Shrinkage techniques use penalty terms to 
automatically perform variable selection. The first lasso will perform the variable selection by using a high penalty coefficient and therefore eliminate many non-significant variables. We then perform the shrinkage step with a second lasso regression. The input variables used in the second regression are not the complete parameter set but the parameters retained by the first lasso. We obtain the optimal penalty parameters by using cross-validation and minimizing the validation error. Double lasso is a shrinkage technique, which is computational efficient, achieves lowbias and has a fast convergence [18]. The most important property from the adaptive lasso is its oracle property, which ensures the consistency of the estimates prediction. More information and mathematical background about the double lasso or relaxed lasso can be found in [18] and in [19] for the adaptive lasso. We kept only the highest significant predictors $(p<0.01 \%)$ and used a five-fold cross-validation to ensure that the regression does not overfit the data.

We need to deal with multicollinearity in order to robustly estimate the regression coefficients. Multicollinearity will lead to a shared variance explanation between highly correlated variables. This can affect the predictive ability of the regression model, as well as the estimation of the regression coefficients and their statistical significance [16]. To avoid multicollinearity between two or more explanatory variables, we look at the VIF. If the VIF exceeds 10, there is an excessive collinearity among the explanatory variables. A VIF between 3 and 5 indicates a serious multicollinearity $[16,20]$. In our study, if the VIF exceeds 4 , we identify the correlated variable which has the lowest statistical significance parameter. We then repeat the generalized regression without this parameter.

\subsection{Prediction of the groove resonance frequency using FEM}

We use the FEM to predict the frequency location of the groove resonance and therefore prove that the noise variation due to the foam insert does correspond to the groove resonance phenomenon. This model is built from footprints made inside the inner drum test bench and the outer geometry of the tire. For the same tire loads used during the tireroad noise measurements, a footprint of each serial tire is made using ink and paper (see the lower part of Fig. 2). We apply an image processing tool to detect the footprint's boundaries and to reduce the edges of the tread blocks to quadratic functions for the summer tire and to spline functions for the winter tire. The footprint is then extruded to a profile height of $8 \mathrm{~mm}$ and fused to the geometry of a deformed tire under stationary rolling condition obtained by in-house FEM computation. The simulation conditions are identical to the testing conditions on the test rig. This geometry is then subtracted from a half-sphere having a diameter of $2 \mathrm{~m}$ in order to have the three-dimensional geometry of the air surrounding the tire. This approach is very similar to the model presented by Fujiwara et al. in [8]. We take into account the drum's curvature by deleting the air volume between the halph sphere's bottom and a cylinder of diameter $3.8 \mathrm{~m}$.
The model was developed using Abaqus 2017. Quadratic acoustic elements build the air surrounding the tire and quadratic infinite elements are added on the half-sphere's curved surface to simulate the free-field condition. The ground and the tire are considered perfectly sound-reflecting. We excite the four circumferential grooves of the summer tire at the leading edge and compute the SPL at the trailing edge. For the winter tire, we excite each groove at the footprint's center and obtain the SPL at the tire sidewalls. For each groove, we obtain a transfer function from $100 \mathrm{~Hz}$ to $3000 \mathrm{~Hz}$ which shows the frequency location of the pipe network's resonances. For each groove, we extract the main resonance frequencies and the corresponding vibration shape.

\section{Results}

\subsection{Overall contribution of groove resonance to tire-road noise}

For both tires, the contribution of groove resonance to tire-road noise averaged over the five microphones for all driving speeds, tire loads and road surfaces equals $1.7 \mathrm{dBA}$. Figure 4 depicts the contribution of groove resonance to tire-road noise around the tire.

The black points are measurements that do not fit within the boxplot's whiskers. This corresponds to a SPL value more than 1.5 times the interquartile range (i.e. 1.5 times the height of the box) away from the bottom or the top of the box. We measured these values at very high speeds on the Asphalt $0 / 5$ or at very low speeds on the SMA $0 / 8$ surface. Although the boxplot representation depicts these measurement points as outliers, we kept them during the statistical analysis. These outliers namely did not affect the global evolution of the groove resonance SPL along the driving speed. As we are interested in the underlying physical relationship between the groove resonance noise and the operating parameters, we considered these outliers as non-critical.

For the summer tire with circumferential grooves the groove resonance is more intensely excited at the leading and trailing edge, and at the tire sidewall's center. For the winter tire the highest groove resonance SPL is observed at the CPX microphones. For the rest of this study we will focus the analysis on the microphones where we measured the highest average groove resonance noise: the trailing edge for the summer tire and the CPX-Middle microphone for the winter tire.

\subsection{Influence of the driving speed and tire load on the groove resonance noise}

The statistical analysis aims at finding the possible interactions between groove resonance noise, operating parameters (tire load and driving speed) and the road surface parameters. First, we perform an analysis on the spectrum and on the groove resonance SPL to estimate the influence of the operating parameters on groove resonance 


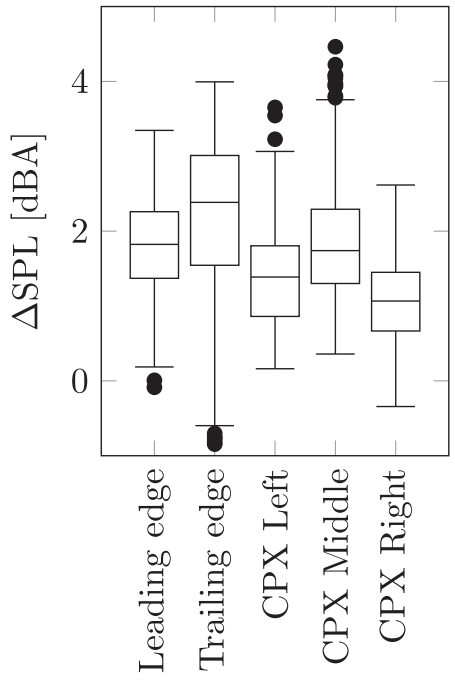

(a) Summer

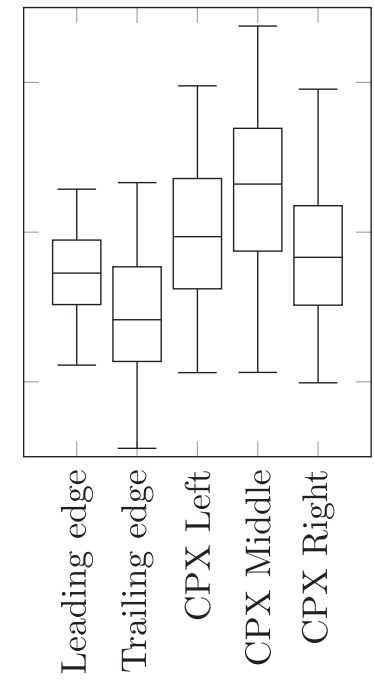

(b) Winter
Figure 4. Averaged contribution of the groove resonance to the tire-road noise on all four road surfaces. The average is computed with all measurements (speeds between 30 and $120 \mathrm{~km} / \mathrm{h}$, loads between 2400 and $3600 \mathrm{~N}$ ).

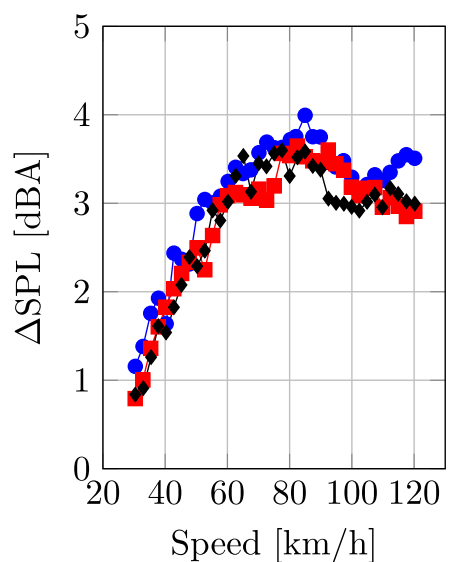

(a) Summer - Trailing

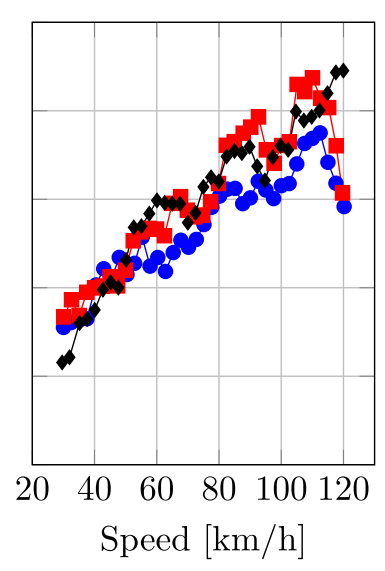

(b) Winter - CPX Middle

$$
\bullet 2400 \mathrm{~N} \longrightarrow 3600 \mathrm{~N} \bullet 4800 \mathrm{~N}
$$

Figure 5. Influence of the tire load and the driving speed on the groove resonance noise level on the Asphalt 0/5.

noise. This will help with the interpretation of the statistical analysis.

The combined influence of tire load and driving speed on the groove resonance noise is depicted in Figure 5. For the summer tire (see Fig. 5a), the slope of the groove resonance SPL declines as the driving speed increases. For speeds above $80 \mathrm{~km} / \mathrm{h}$, the groove resonance SPL decreases for all tire loads. For the winter tire (see Fig. 5b), the groove resonance SPL increases on a linear scale with driving speed. The influence of tire load on the groove resonance SPL is not clear. It seems that a lower tire load will increase the groove resonance for the summer tire, while no clear

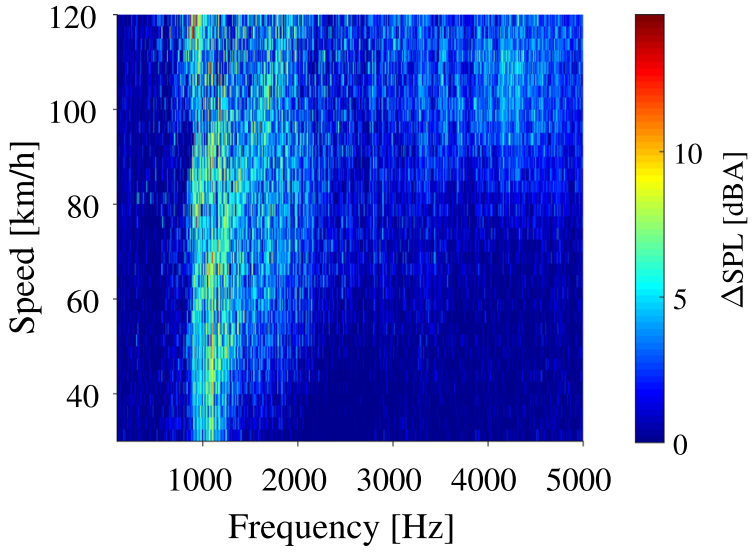

(a) $2400 \mathrm{~N}$

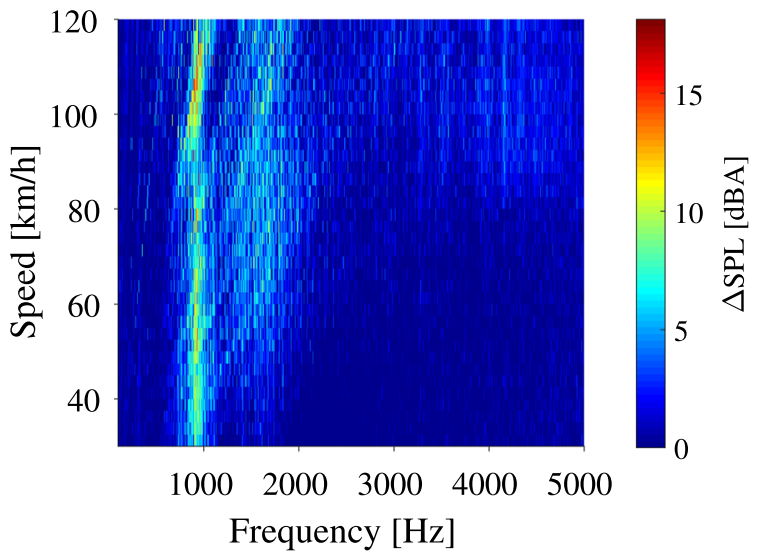

(b) $3600 \mathrm{~N}$

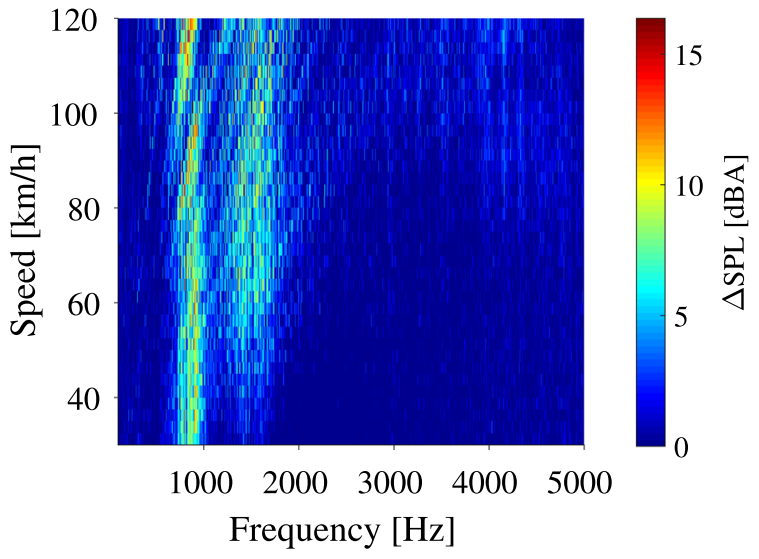

(c) $4800 \mathrm{~N}$

Figure 6. Spectral analysis of the groove resonance noise of the summer tire on the asphalt $0 / 5$ at the trailing edge microphone.

tendency can be seen for the winter tire. The curve's slope in Figure 5b seems to be depending upon the tire load, which could lead to an interaction between the tire load and the driving speed for the winter tire.

The combined influence of driving speed and tire load on the spectrum of $\triangle \mathrm{SPL}$ is depicted in Figure 6 for the summer tire and in Figure 7 for the winter tire. The vertical lines in the spectra correspond to a phenomenon with a 


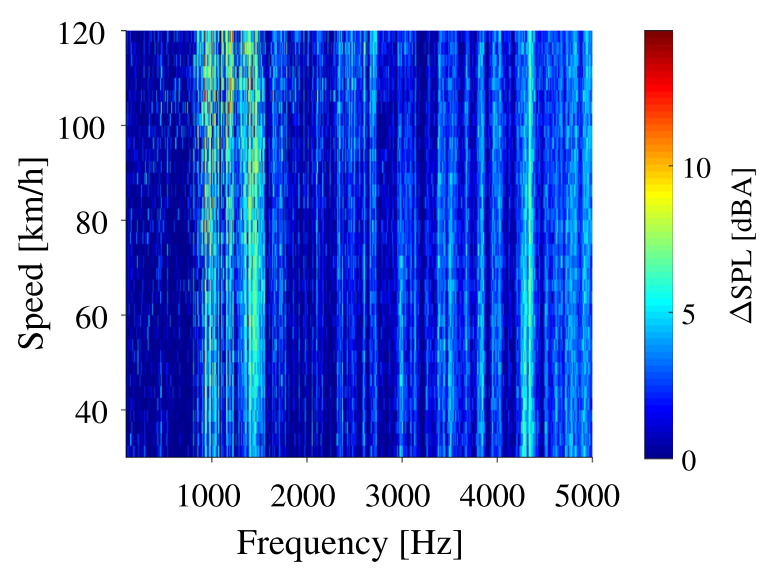

(a) $2400 \mathrm{~N}$

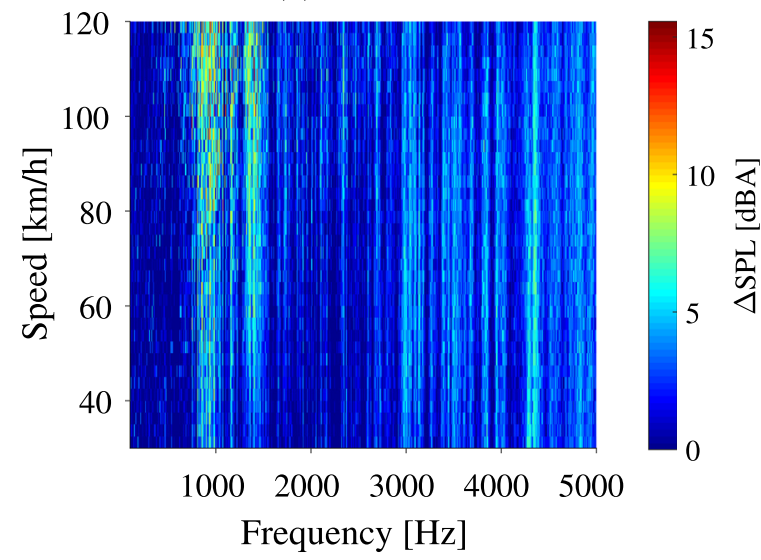

(b) $3600 \mathrm{~N}$

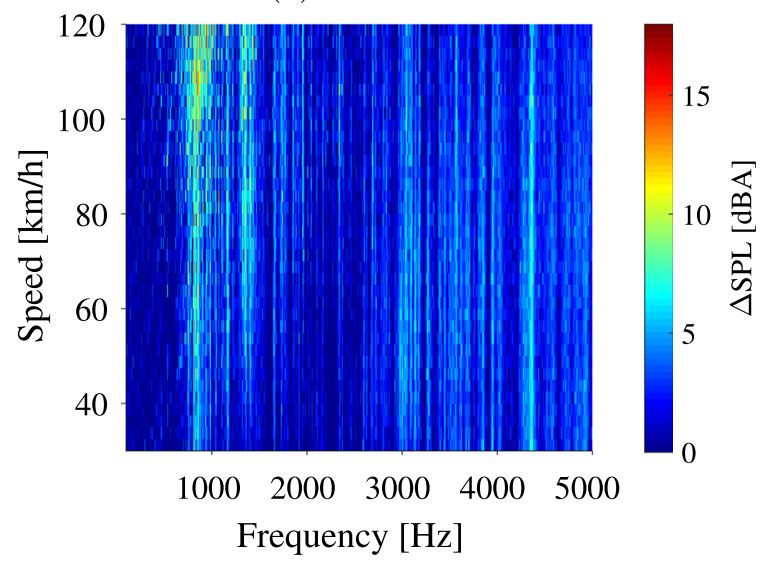

(c) $4800 \mathrm{~N}$

Figure 7. Spectral analysis of the groove resonance noise of the winter tire on the asphalt $0 / 5$ at the CPX-Middle microphone.

frequency depending on the driving speed. They therefore depict the groove resonance contribution to tire-road noise. A light speed-dependent contribution can be seen in the summer tire's spectrum (see Fig. 6). They correspond to the tread impact noise, which should not be seen if the foam had no influence on the tread block's flexibility. It seems that the tread impact mechanism is slightly affected by the foam inside the grooves. However, it seems that groove resonance is the main phenomenon contributing to $\triangle \mathrm{SPL}$.
Table 2. Influence of the tire load on the footprint length and the theoretical pipe resonance frequencies. The frequency shift in percent is given relatively to the resonance frequency at $3600 \mathrm{~N}$.

\begin{tabular}{lccc}
\hline & $2400 \mathrm{~N}$ & $3600 \mathrm{~N}$ & $4800 \mathrm{~N}$ \\
\hline Groove length [mm] & 88 & 110 & 133 \\
Resonance frequency [Hz] & 1949 & 1681 & 1289 \\
Frequency shift [\%] & +15.9 & 0 & -23.3 \\
\hline
\end{tabular}

Although tire load seems to have a relatively low influence on the groove resonance SPL, it has an influence on the spectral content of the groove resonance noise. As shown in Figure 6, a higher tire load will lead to a longer contact patch, which results in a decreased groove resonance frequency. This fundamental frequency lies around $1040 \mathrm{~Hz}$ for a tire load of $2400 \mathrm{~N}$ and around $840 \mathrm{~Hz}$ if we double the tire load. A higher tire load also leads to a more defined excitation of the second groove resonance harmonic (see second vertical line around $1700 \mathrm{~Hz}$ in Fig. 6c).

The influence of tire load on the groove resonance spectrum of the winter tire is similar (see Fig. 7). We observe a reduction of the fundamental groove resonance frequency from 1000 to $800 \mathrm{~Hz}$ by increasing the tire load from 2400 to $4800 \mathrm{~N}$. Due to the complex V-shaped geometry of the winter tread pattern (see Fig. 2d), we have different groove lengths which contribute to the groove resonance noise. This explains the complexity of the groove spectrum which shows spectral components above $3000 \mathrm{~Hz}$.

A comparison with the pipe resonance theory is needed to confirm the influence of tire load on the frequency shift of the groove resonances. This is summarized in Table 2 for the summer tire. A large gap is observed between the measured and the theoretical groove resonance frequencies. In average, the measured frequencies are $42 \%$ lower than the computed ones. The computed resonance frequencies correspond to the groove length of the contact patch. The influence of the groove network, the drum curvature and the horn geometry of the deformed tire is not taken into account. This will be done using a numerical approach in the next section. Due to the complexity of the winter tire's profile, we did not realize any prediction of its groove resonance frequencies.

The suppositions enounced above need to be confirmed. Analyzing the spectrum of $\triangle \mathrm{SPL}$ along the speed showed that inserting foam into the tire's grooves might affect the tread pattern noise and maybe also the belt vibrations. We will show in the next section that the measured SPL variation mainly corresponds to groove resonance noise.

\subsection{FEM results of the groove resonance vibration modes and frequencies}

The main resonance modes for both tires are depicted in Figure 8. For the winter tire, it consists in a transversal flexion mode of the complete footprint (see Fig. 8a). Another major resonance is the individual resonance of each groove 


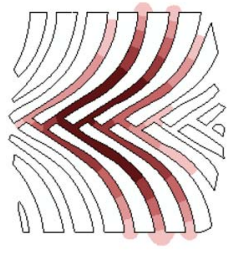

(a)

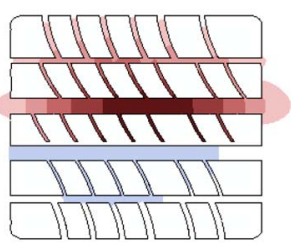

(c)

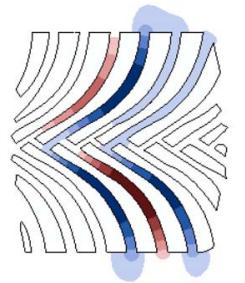

(b)

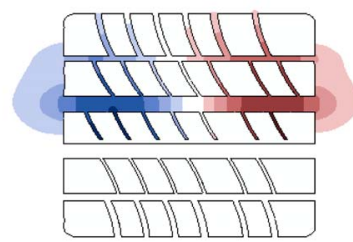

(d)
Figure 8. Main resonance shapes computed inside the inner drum test bench with a tire load of $3600 \mathrm{~N}$.

(see Fig. 8b). For the summer tire, the footprint can be divided into two parts, each of them consisting of two circumferential grooves connected through transversal grooves and ventilated towards the tire's sidewall. Each part has a fundamental resonance frequency (see Fig. 8c for the upper half with resonators). This longitudinal flexion mode also has a second harmonic (see Fig. 8d).

We now extract these frequencies for all tire loads and compare them to the measured groove resonance spectrum. As we showed in Figures 6 and 7, the frequency content of the groove resonance noise is not dependent on the driving speed. We therefore compute the average spectrum over the speed to facilitate the comparison between measurement and simulation. This is depicted in Figures 9 and 10, respectively for the summer and the winter tire. For the summer tire under all tire loads, the computed frequency location of the first and the second groove resonances corresponds perfectly to the measured SPL peaks. Although the simulation method takes place in static conditions, it seems that the groove resonance phenomenon is adequately built for the summer tire. This also confirms the hypothesis that the foam insert has an influence mainly on the groove resonance phenomenon, at least for the summer tire.

The results for the winter tire show a good agreement between the computed and the measured groove resonance frequencies. In average, the model tends to overestimate the resonance frequencies by $175 \mathrm{~Hz}$ for the lowest tire load and by $95 \mathrm{~Hz}$ for the tire loads of $3600 \mathrm{~N}$ and $4800 \mathrm{~N}$. This could be due to the simplifications realized to obtain the numerical model. Comparing the winter tire's footprint from Figure 2d and the corresponding resonance modes of Figure 8 shows that the lamellae are not taken into account inside the calculation. Including the lamellae into the model would lead to a higher connectivity between the different grooves and therefore reduce the groove resonance frequencies of the winter tire. The experimental results show a high influence of the foam insert on the noise above $2000 \mathrm{~Hz}$ (see Fig. 7), which the model does not explain.
This high-frequency noise could correspond to the airpumping noise which could be affected by the foam insert.

\subsection{Influence of the road surface on the groove resonance noise}

The retained parameters by the double lasso are listed in decreasing significance order in Table 3 for the summer tire and in Table 4 for the winter tire. The regression equation can be computed by summing over the table lines the product of the "Term" and "Estimate" columns. For the training and testing data, the coefficient of determination $R^{2}$ is $92 \%$ and the RMSE lies around $0.3 \mathrm{dBA}$.

For both tires, the driving speed is the factor having the highest significance. For the summer tire, the speed influence is declining with increasing speed (positive estimate of Speed and negative estimate of Speed ${ }^{2}$ ), while the speed influence is linear for the winter tire. This confirms the results obtained in Figure 5. The evolution of the groove resonance noise along the speed for the summer tire namely shows a turning point at speeds around $80 \mathrm{~km} / \mathrm{h}$. On the contrary, the SPL curves of the winter tire curves seem to increase linearly with the driving speed.

The most significant road surface parameters are also identical for both tires and can be divided into two groups: the roughness parameters and the absorption parameters. Indeed the interaction between the roughness amplitude MPD and its orientation RSK is the most significant parameter describing a road surface property. On the other hand, the logarithm of the absorption coefficient also has a high significance on the groove resonance. It seems that the texture orientation is an important parameter for the groove resonance noise, as it appears for both tires within the four most significant parameters.

Several interactions are highly significant. For the summer tire, the absorption's influence is high at lower speeds and decreases with increasing speed. We also observe an interaction between the tire load and the texture orientation RSK. A higher load combined with a positive oriented texture has the same effect on groove resonance as a lower load on a negative oriented texture: an increase of the groove resonance noise. The other two combinations lead to a decrease in groove resonance noise. The interaction between the load and the speed indicates that the speed's leverage increases with increasing load. The speed will increase groove resonance noise more at higher loads than at lower tire loads. The same observation can be made with the interaction between the tire load and the MPD. Although these interactions are highly significant, they only influence the regression's coefficient of determination by $2 \%$ and decrease the RMSE by $0.04 \mathrm{dBA}$.

The relative influence of each parameter on the regression's coefficient of determination and RMSE is depicted in Figure 11. A number of parameters of zero corresponds to the Intercept model. We then add parameters in a decreasing order of significance according to Tables 3 and 4. The interaction of MPD with RSK has the highest influence on the quality of fit. It reduced the RMSE by 


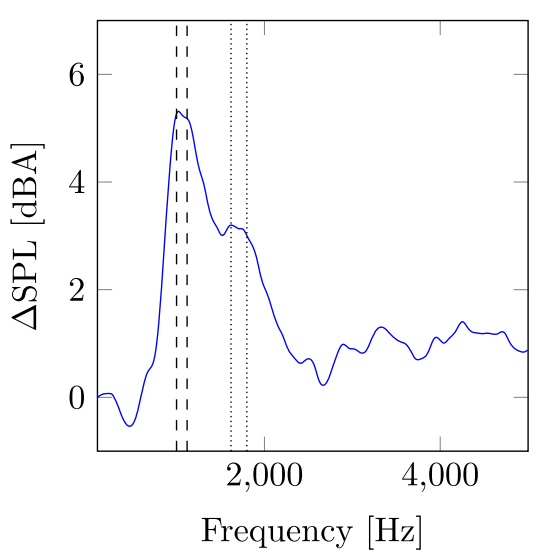

(a) $2400 \mathrm{~N}$

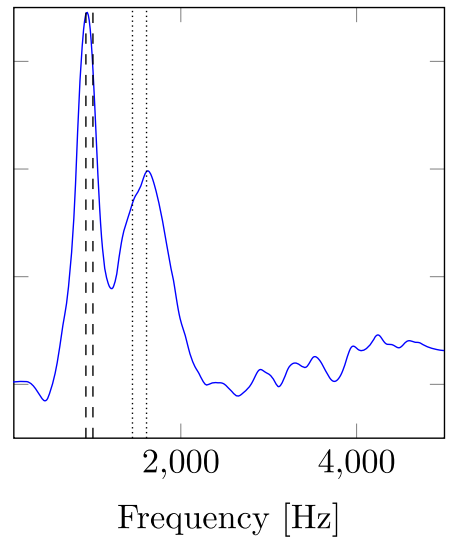

(b) $3600 \mathrm{~N}$

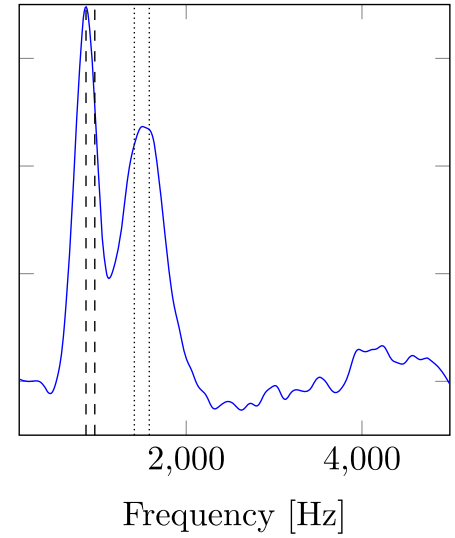

(c) $4800 \mathrm{~N}$

Figure 9. Comparison between the measured groove resonance's spectrum and the computed groove resonance frequencies of the summer tire. The dashed lines correspond to the first resonance of the inner grooves (see Fig. 8c). The dotted lines depict the second resonance of the inner grooves (see Fig. 8d).

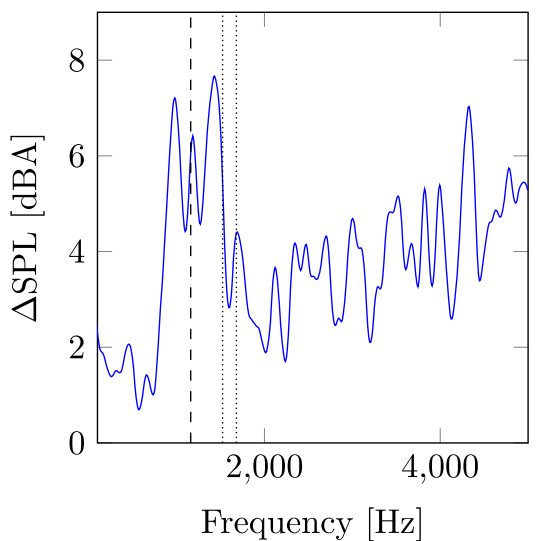

(a) $2400 \mathrm{~N}$

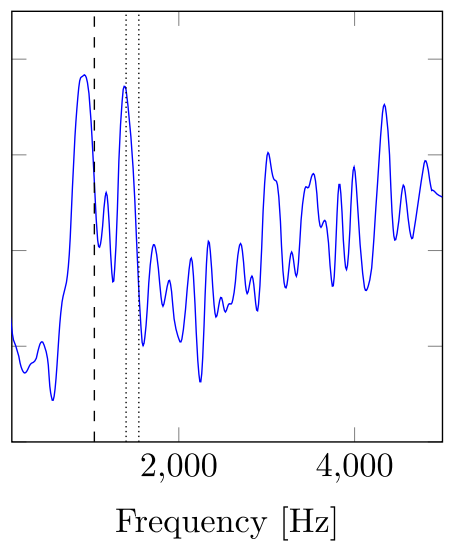

(b) $3600 \mathrm{~N}$

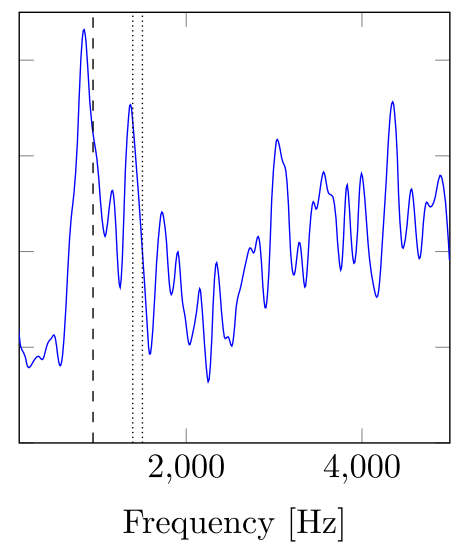

(c) $4800 \mathrm{~N}$

Figure 10. Comparison between the measured groove resonance's spectrum and the computed groove resonance frequencies of the winter tire. The dashed line corresponds to the transverse resonance of the footprint (see Fig. 8a). The dotted lines depict the resonance frequency boundaries of the winter tire's grooves (see Fig. 8b)

Table 3. Regression results of the summer tire. The first column corresponds to the $x$-axis of Figure 11.

\begin{tabular}{|c|c|c|c|c|}
\hline$\#$ & Term & Estimate $(95 \% \mathrm{CI})$ & Wald $\chi^{2}$ & Prob $>\chi^{2}$ \\
\hline 2 & $(\mathrm{MPD}-1.04) \times(\mathrm{RSK}+0.59)$ & $4.756(4.334,5.178)$ & 488.6 & $<.0001$ \\
\hline 3 & $(\text { Speed }-75.2)^{2}$ & $-3.33 \mathrm{e}-4(-3.85 \mathrm{e}-4,-2.81 \mathrm{e}-4)$ & 155.8 & $<.0001$ \\
\hline 4 & $($ Load -3600$) \times($ RSK +0.59$)$ & $3.283 \mathrm{e}-4(2.738 \mathrm{e}-4,3.828 \mathrm{e}-4)$ & 139.3 & $<.0001$ \\
\hline 5 & $\log (\alpha)$ & $-0.504(-0.616,-0.392)$ & 77.2 & $<.0001$ \\
\hline 6 & $($ Speed -75.2$) \times(\log (\alpha)+1.53)$ & $9.481 \mathrm{e}-3(6.057 \mathrm{e}-3,1.291 \mathrm{e}-2)$ & 29.5 & $<.0001$ \\
\hline & Training set & \multicolumn{2}{|l|}{0.92} & 0.30 \\
\hline & Validation set & \multicolumn{2}{|l|}{0.92} & 0.30 \\
\hline
\end{tabular}


Table 4. Regression results of the winter tire. The first column corresponds to the $x$-axis of Figure 11.

\begin{tabular}{|c|c|c|c|c|}
\hline$\#$ & Term & Estimate $(95 \% \mathrm{CI})$ & Wald $\chi^{2}$ & Prob $>\chi^{2}$ \\
\hline 1 & Speed & $0.0245(0.0231,0.0259)$ & 1196.1 & $<.0001$ \\
\hline 2 & Load & $3.229 \mathrm{e}-4(2.896 \mathrm{e}-4,3.561 \mathrm{e}-4)$ & 362.0 & $<.0001$ \\
\hline 3 & $(\mathrm{MPD}-1.04) \times(\mathrm{RSK}+0.59)$ & $3.407(3.039,3.775)$ & 329.7 & $<.0001$ \\
\hline 4 & RSK & $0.383(0.322,0.444)$ & 151.7 & $<.0001$ \\
\hline 5 & $($ Load -3600$) 2$ & $-2.429 \mathrm{e}-7(-2.920 \mathrm{e}-7,-1.939 \mathrm{e}-7)$ & 94.3 & $<.0001$ \\
\hline 0 & Intercept & $-1.028(-1.307,-0.750)$ & 52.3 & $<.0001$ \\
\hline 6 & $\log (\alpha)$ & $-0.504(-0.649,-0.360)$ & 46.7 & $<.0001$ \\
\hline \multirow[t]{4}{*}{8} & $($ Load -3600$) \times($ Speed -75.2$)$ & $3.579 \mathrm{e}-6(1.894 \mathrm{e}-6,5.264 \mathrm{e}-6)$ & 17.3 & $<.0001$ \\
\hline & & $R^{2}$ & & RMSE $[\mathrm{dBA}$ \\
\hline & Training set & 0.92 & & 0.30 \\
\hline & Validation set & 0.92 & & 0.32 \\
\hline
\end{tabular}

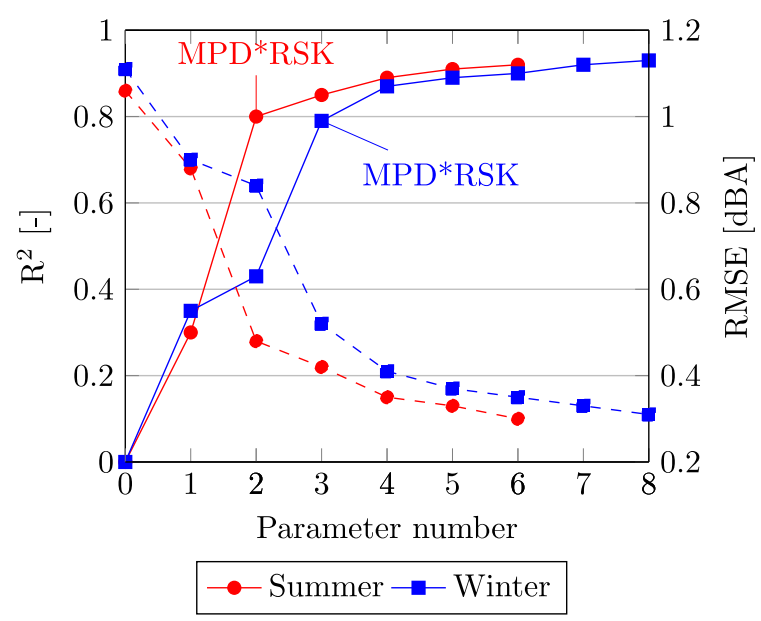

Figure 11. Influence of the number of parameters on the multiple linear regression's quality of fit. The plain lines correspond to the coefficient of determination $R^{2}$. The dashed lines depict the RMSE. The parameter number corresponds to the first column of Tables 3 and 4.

$45 \%$ and $38 \%$ while increasing the coefficient of determination by $50 \%$ and $36 \%$ respectively for the summer and winter tire.

\section{Discussion}

The distribution of the groove resonance's SPL along the footprint (see Fig. 4) could be expected. We expect namely the circumferential grooves of the summer tire to radiate noise at their opened ends: the leading and trailing edges. The relatively high groove resonance noise at the CPX-Middle microphone can be explained with the transversal grooves. The resonance antinode is namely located at the center of the contact patch. Groove resonance will therefore be ventilated outside the contact patch through the transversal grooves. Since the CPX-Middle microphone is located at the tire's sidewall, towards the middle of the contact patch, observing a high contribution of the groove resonance noise at this location is logical.
For the winter tire with $\mathrm{V}$-shaped grooves, the grooves open up at the tire's sidewalls, which explains the high groove resonance noise at the CPX microphones.

The measured contribution of groove resonance noise to tire-road noise is approximately half of what Saemann measured in [4]. However, the comparison in Saemann's study was made between a slick and a ribbed tire so the influence of tread pattern noise is not taken into account. Moreover, the results from Saemann were realized on an outer drum. Although this is not specified within the paper, we suppose they made the measurements on the same test rig presented in [21], which uses a smooth asphalt replica. This replica should have a MPD in the same range as our smooth Asphalt 0/5. If we compare their results with our measurements realized on the Asphalt 0/5, we also observe an average groove resonance contribution to tire-road noise of $2.9 \mathrm{dBA}$. For a nominal tire width of $205 \mathrm{~mm}$, the newest version of the R117 regulation imposes a reduction of tire rolling noise by $4 \mathrm{dBA}$ compared to the previous regulation [22]. Although the measurement procedure for the tire approval test is different, reducing groove resonance noise could be a very efficient way to reduce tire-road noise and meet the new regulation requirements. Inserting foam into the grooves is not a viable option but several alternatives have already been proposed such as using subresonators [8] or by optimizing the ventilation of the pipe network [9].

The reduction of the groove resonance frequency with increasing tire load is also logical. A higher load leads to a longer contact patch and therefore to a longer groove length. For the winter tire with V-shaped grooves, an increase of tire load also leads to a wider contact patch which also increases the groove length. The non-linear increase of groove resonance noise in Figure 5 a can be explained by the part of Figure 6 around 80 and $100 \mathrm{~km} / \mathrm{h}$. It seems that there is a discontinuity along the speed of the groove resonance contribution. This is especially visible on Figure $6 \mathrm{c}$ at $100 \mathrm{~km} / \mathrm{h}$. This could be explained by a relative higher contribution of tread impact noise to tire-road noise. Unlike the groove resonance noise, the spectral content of the tread pattern noise depends on the driving speed. There are speeds where the tread pattern noise harmonics will cross the groove resonance frequency which are critical in terms of tire rolling 
noise emission [2]. We believe that the interaction between tread pattern noise harmonics and the groove resonance amplifies the noise level around $80 \mathrm{~km} / \mathrm{h}$. The tread pattern vibrations could here act as an excitation source for the groove resonance. As the tread pattern noise harmonics does not coincide with the groove resonance around $100 \mathrm{~km} / \mathrm{h}$, the discontinuity seen in Figure 6c could appear. This could explain the reduction of the groove resonance SPL for the summer tire around $100 \mathrm{~km} / \mathrm{h}$ (see inflexion points at these speeds on Fig. 5a).

The results of the regression analysis agrees well with the observations presented in Section 3.2 for the operating parameters influence on the groove resonance SPL. Driving speed is the parameter having the highest significance on the groove resonance noise. Its influence is linear for the winter tire and quadratic for the summer tire. The negative estimate of Speed $^{2}$ in Table 3 explains the reduction of groove resonance noise for the summer tire at speeds around $80 \mathrm{~km} / \mathrm{h}$ in Figure $5 \mathrm{a}$. Tire load is also highly significant for the winter tire, as increasing the tire load will lead to a longer contact patch and therefore to a bigger number of potentially resonating grooves. The interaction between the tire load and the driving speed for the winter tire helps explaining the slope difference in Figure 5b.

An interesting result is that the MPD itself has no influence on the groove resonance noise. This parameter is used with the acoustic absorption to describe the test tracks used for tire approval tests [23]. For the parameters describing the road surface characteristics, the interaction between the roughness amplitude and its orientation is the parameter having the highest influence on groove resonance noise. The influence of this interaction is showed in Figure 12. Groove resonance is increased on:

- A smooth negative oriented surface;

- A rough positive oriented surface.

The first case (lower-left part of Fig. 12) can be explained by a better sealing of the tire on the road. The road surface is too fine to offer connected voids and the resonance is more likely to take place. It is hard to explain the groove resonance's increase with a rough positive oriented surface (upper-right part of Fig. 12). One could expect that the pipe network is not completely closed due to the high macro-roughness. This should lead to a lesser excitation of the pipe resonance. This could however lead to a higher excitation of the tire structural vibrations and to a reduction of tread impact noise. This will shift the tire-road noise frequency peak to lower frequencies. The groove resonance becomes consequently the main noise contributor between 800 and $1000 \mathrm{~Hz}$.

The last two combinations lead to a decrease of groove resonance noise. A rough negative oriented texture (lowerright corner of Fig. 12) means the road surface is flat with voids, which might be connected with one another. This will consequently lead to a non-hermetic seal between the tire and the road, which explains a lesser excitation of the groove resonance. It is difficult to interpret the upper-left

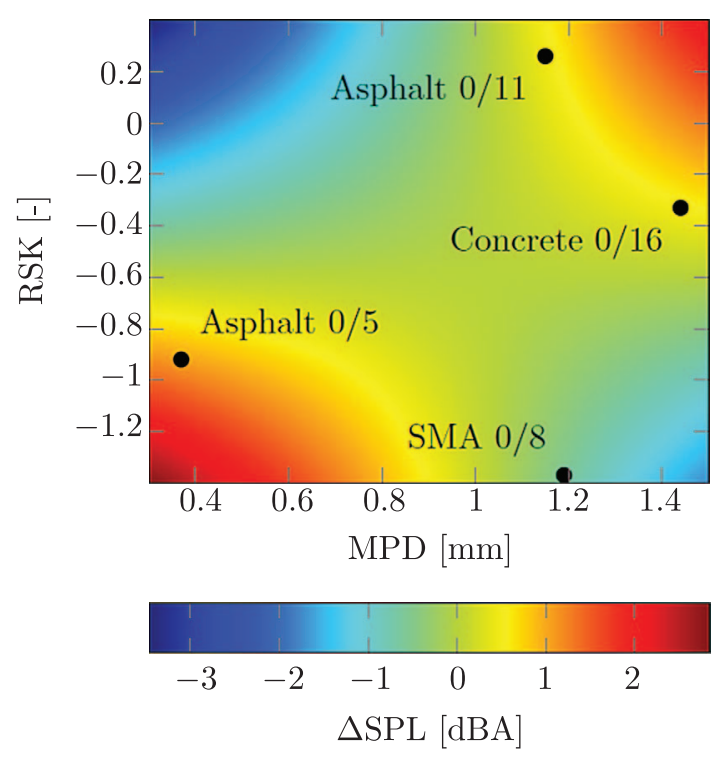

Figure 12. Influence of the interaction between the roughness amplitude MPD and the texture orientation RSK on the summer tire's groove resonance noise. The result for the winter tire is similar but the SPL range is reduced by $28 \%$. The black points correspond to the measurement points.

corner of Figure 12, especially because the tested road surfaces do not cover this range of the MPD $\times$ RSK interaction. As we do not have measurement points to validate this corner, the decrease of groove resonance noise on a smooth positive road surface might be due to the extrapolation of the statistical results.

We expect an identical influence of the acoustic absorption on the groove resonance noise, independently of the groove geometry. Absorption mainly influences the noise radiation and always absorb the same amount of groove resonance noise. It is confirmed by the similar estimate value of $\log (\alpha)$ in Tables 3 and 4 .

\section{Conclusion}

We have presented a method to identify the relevant parameters influencing the groove resonance contribution to tire-road noise. By filling the tire grooves with acoustic foam, we are able to isolate the groove resonance noise from the tire rolling noise. We quantified the influence of operating parameters and road surface descriptors on groove resonance noise by using multiple linear regression. For all measurements, we note a groove resonance noise contribution to tire-road noise of about $1.7 \mathrm{dBA}$ in average. This contribution depends mainly upon the driving speed, the tire load and the road surface characteristics. While the above mentioned parameters are mainly influencing the amplitude of the groove resonance noise, the tread pattern design as well as the tire load modify the spectral content of the groove resonance. We showed that the MPD alone is not a relevant parameter to characterize the road surface's influence on 
groove resonance noise. The interaction between MPD and RSK is the most significant parameter related to road surface descriptors. The absorption coefficient is also another significant parameter.

We only tested two tires that represent the generic forms of tread pattern we can find on the market: tires with circumferential grooves and tires with V-Shaped grooves. A more extensive measurement plan should be done with more tires to ensure the robustness of the results. We also studied only dense asphalts with an absorption coefficient lower than $15 \%$. Including porous asphalts in the analysis would be another step to cover all types of asphalts used in civil construction. We considered that the acoustic foam absorbed completely the groove resonance noise. This hypothesis is limited by the absorption spectrum of the foam which we can not expect to reach $100 \%$ at frequencies between 800 and $1000 \mathrm{~Hz}$. Moreover, we did not fill the transversal grooves of the summer tire. We therefore suppose that the contribution of groove resonance noise to tire-road noise could be higher than in the measurements presented in this study.

The hypothesis that only groove resonance is affected by the foam insert is questionable. Although the numerical results provides good evidence that the foam insert does affect the groove resonance noise, it could affect also the vibroacoustical behavior of the tread blocks as well as the air pumping phenomenon.

The loading condition on an inner drum is also different as on a flat surface. Due to the drum curvature, the contact patch will be longer and the groove resonance frequency will therefore be underestimated. The influence of the drum curvature could be studied by testing the same tires on different tracks with a CPX-trailer. The numerical approach presented in this study could also help assessing the drum curvature's influence on the groove resonance frequency.

Our findings can be used by tire manufacturers to optimize their tread design. It seems that groove resonance becomes a significant noise source on smooth negative oriented road surfaces. We suppose that the standardized tracks for tire approval tests correspond to this description. Groove resonance noise also increases at speeds between 70 and $90 \mathrm{~km} / \mathrm{h}$, which are relevant speeds for tire approval tests. Optimizing the tread design for groove resonance could reduce tire-road noise by several decibels and help fulfilling the upcoming decrease of tire-road noise limits.

This study can also be the first step towards a review of the ISO-10844 standard. As the MPD is the standardized parameter used to describe test tracks for tire approval test, it would be interesting to study the interaction between the roughness amplitude and the texture orientation on tire rolling noise levels. The texture orientation could namely be an important parameter overseen by the ISO-10844 standard. Due to the place limitation on the inner drum test rig, we could perform the measurements only in near-field conditions. Performing the same measurements in far-field conditions would be the next step to study the influence of road surface characteristics on groove resonance noise radiation.

\section{Nomenclature}

$\begin{array}{ll}\text { CPX } & \text { Close-Proximity } \\ \text { FEM } & \text { Finite Element Method } \\ \text { MLR } & \text { Multiple Linear Regression } \\ \text { MPD } & \text { Mean Profile Depth } \\ \text { RMS } & \text { Root Mean Square } \\ \text { RMSE } & \text { Root Mean Square Error } \\ \text { RSK } & \text { Skewness } \\ \text { RKU } & \text { Kurtosis } \\ \text { SPL } & \text { Sound Pressure Level } \\ \text { VIF } & \text { Variance Inflation Factor }\end{array}$

\section{Conflict of interest}

Author declared no conflict of interests.

\section{References}

1. M. Dittrich, F. De Roo, S. Van Zyl, S. Jansen, E. De Graaff, J. Sliggers: Triple a tyres for cost-effective noise reduction in Europe, 10th European Congress and Exposition on Noise Control Engineering EuroNoise 2015 (2015) 2607-2612.

2. U. Sandberg, J. Ejsmont: Tyre/road noise-Reference Book, Informex, Kisa, 2002.

3. B. Favre: Some results concerning current research on tire noise in France, International Tire Noise Conference, Stockholm, 1979.

4. E.-U. Saemann, H. Schmidt: Schallmessungen bei der entwicklung von reifen mit geringem vorbeifahrtpegel, Zeitschrift für Lärmbekämpfung 49 (2002) 59-62.

5. I.D. Wilken, L.J. Oswald, R. Hickling: Research on individual noise source mechanisms of truck tires: Aeroacoustic sources, in: SAE Technical Paper Series. SAE International, Commonwealth Drive, Warrendale, PA, United States (1976).

6. J. Ejsmont, U. Sandberg, S. Taryma: Influence of tread pattern on tire/road noise, in Passenger Car Meeting \& Exposition, SAE Technical Paper Series. SAE International, Commonwealth Drive, Warrendale, PA, United States (1984).

7. T. Fujikawa, H. Koike, Y. Oshino, H. Tachibana: Generation mechanism of tire/road noise. Part 2: Pipe resonance in tread groove of tire, in: Proceedings of Inter-Noise 99. J. Cuschieri, S. Glegg, Y. Yong, Editors, 1999.

8. S. Fujiwara, K. Yumii, T. Saguchi, K. Kato: Reduction of tire groove noise using slot resonators, Tire Science and Technology 37 (2009) 207-223.

9. B. Wang, D. Duhamel: On the design and optimization of acoustic network resonators for tire/road noise reduction, Applied Acoustics 120 (2017) 75-84.

10. R. Gnadler, H.-J. Unrau, M. Frey, M. Fertig: Grundsatzuntersuchung zum quantitativen Einfluß von Reifenbauform und -ausführung auf die Fahrstabilität von Kraftfahrzeugen bei extremen Fahrmanövern, volume 192 of FATSchriftenreihe, Forschungsvereinigung Automobiltechnik e.V., Frankfurt, M., 2005.

11. ISO 11819-2: Acoustics - measurement of the influence of road surfaces on traffic noise - part 2: The close-proximity method (2017).

12. ISO 13473-1: Characterization of pavement texture by use of surface profiles - part 1: Determination of mean profile depth (2017). 
13. ISO 13473-2: Characterization of pavement texture by use of surface profiles - part 2: Terminology and basic requirements related to pavement texture profile analysis (2002).

14. G. Bitelli, A. Simone, F. Girardi, C. Lantieri: Laser scanning on road pavements: A new approach for characterizing surface texture, Sensors 12 (2012) 9110-9128.

15. ISO 13472-2: Acoustics - measurement of sound absorption properties of road surfaces in situ - part 2: Spot method for reflective surfaces (2010).

16. J.F. Hair, W.C. Black, B.J. Babin, R.E. Anderson: Multivariate data analysis, 8th edn., Cengage Learning EMEA, Andover, Hampshire, 2019.

17. D.C. Montgomery, E.A. Peck, G. Geoffrey Vining: Introduction to linear regression analysis, in volume 821 of Wiley Series in Probability and Statistics, 5th edn., Wiley, Hoboken, NJ, 2012.

18. N. Meinshausen: Relaxed lasso, Computational Statistics and Data Analysis 52 (2007) 374-393.
19. H. Zou: The adaptive lasso and its oracle properties, Journal of the American Statistical Association 101 (2006) 1418 1429.

20. C.B. García, J. García, M.M. López Martín, R. Salmerón: Collinearity: revisiting the variance inflation factor in ridge regression, Journal of Applied Statistics 42 (2015) 648-661.

21. Verbundprojekt: "Straßenverkehr 2": Reduzierte ReifenFahrbahn-Geräusche, in: volume 74 of Berichte der Bundesanstalt für Strassenwesen S, Strassenbau. Techn. Informationsbibl. und Univ.-Bibl and Wirtschaftsverl. NW Verl. für neue Wiss, Hannover and Bremerhaven, 2012.

22. UNECE. Regulation No. 117: Uniform provisions concerning the approval of tyres with regard to rolling sound emissions and/or to adhesion on wet surfaces and/or to rolling resistance, February 2016.

23. ISO 10844: Acoustics - specification of test tracks for measuring noise emitted by road vehicles and their tyres (2014).

Cite this article as: Pinay J, Saito Y, Mignot C \& Gauterin F. 2020. Understanding the contribution of groove resonance to tireroad noise on different surfaces under various operating conditions. Acta Acustica, 4, 6 . 